\title{
Closed incompressible surfaces in the complements of posi- tive knots
}

Makoto Ozawa

This paper is dedicated to Professor Shin'ichi Suzuki for his 60th birthday

\begin{abstract}
We show that any closed incompressible surface in the complement of a positive knot is algebraically non-split from the knot, positive knots cannot bound non-free incompressible Seifert surfaces and that the splittability and the primeness of positive knots and links can be seen from their positive diagrams.
\end{abstract}

Mathematics Subject Classification (2000). 57M25.

Keywords. Positive knot, closed incompressible surface, order, free Seifert surface, splittability, primeness.

\section{Introduction}

A knot $K$ in the 3 -sphere $S^{3}$ is called positive if it has an oriented diagram all crossings of which are positive crossings. For a closed surface $F$ in $S^{3}-K$, we define the order $o(F ; K)$ of $F$ for $K$ as follows $([5])$. Let $i: F \rightarrow S^{3}-K$ be the inclusion map and let $i_{*}: H_{1}(F) \rightarrow H_{1}\left(S^{3}-K\right)$ be the induced homomorphism. Since $\operatorname{Im}\left(i_{*}\right)$ is a subgroup of $H_{1}\left(S^{3}-K\right)=\mathbb{Z}\langle$ meridian $\rangle$, there is an integer $m$ such that $\operatorname{Im}\left(i_{*}\right)=m \mathbb{Z}$. Then we define $o(S ; K)=m$.

The positive knot complements have the following special properties.

Theorem 1.1. Any closed incompressible surface in a positive knot complement has non-zero order.

A Seifert surface $F$ for a knot is said to be free if $\pi_{1}\left(S^{3}-F\right)$ is a free group. In [5, Theorem 1.1], it is shown that a knot bounds a non-free incompressible Seifert surface if and only if there exists a closed incompressible surface in the

Partially supported by Fellowship of the Japan Society for the Promotion of Science for Japanese Junior Scientists. 
knot complement whose order is equal to zero. Therefore, Theorem 1.1 gives us the next corollary.

Corollary 1.2. Positive knots cannot bound non-free incompressible Seifert surfaces.

Although positive links which have connected positive diagrams are non-split because they have positive linking numbers, we can give another geometrical proof of this fact.

Theorem 1.3. Positive links are non-split if their positive diagrams are connected.

Positive diagrams of positive knots or links also tell us their primeness. We say that a knot or link diagram $\tilde{K}$ on the 2-sphere $S$ is prime if for any loop $l$ in $S$ intersecting $\tilde{K}$ in 2 points, $l$ bounds a disk intersecting $\tilde{K}$ in an arc.

Theorem 1.4. Non-trivial positive knots or links are prime if their positive diagrams are connected and prime.

Remark 1.5. The referee suggested that one can show that: A non-trivial positive link is prime iff its positive diagram is connected and prime, with the addition of the assumption that the positive link projections contain no nugatory crossings. In fact, the converse of Theorem 1.3 and 1.4 is true, but it needs [2, Theorem 3].

There are other results about determining when a link projection represents a non-split or prime link.

For the splittability,

- alternating links ([1, Theorem 10.2], [4, Theorem 1 (a)]);

- almost alternating links $([6])$;

- homogeneous links ([2, Corollary 3.1]).

For the primeness,

- alternating links ([4, Theorem $1(\mathrm{~b})])$;

- positive braids ([3, 1.2 Theorem $])$.

\section{Proof of Theorem 1.1 and 1.3}

Theorem 1.1 and 1.3 follow from the next Theorem.

Theorem 2.1. Let $K$ be a positive knot or link in the 3-sphere $S^{3}$ and $F$ a closed incompressible surface in the complement of $K$. Then one of the following conclusions hold. 
(1) There exists a loop $l$ in $F$ such that $l k(l, K) \neq 0$.

(2) $F$ is a splitting sphere for $K$, and any positive diagram of $K$ is disconnected.

Henceforth, we shall prove Theorem 2.1 .

Let $S$ be a 2 -sphere in $S^{3}$ and $p: S^{3}-\{2$ points $\} \cong S \times R \rightarrow S$ a projection. Put $K$ so that $p(K)$ is a positive diagram. As usual way, we express $K$ in a bridge presentation. Thus we have the following data (see Figure 1).

- $S^{3}=B^{+} \cup_{S} B^{-}\left(S\right.$ decomposes $S^{3}$ into two 3-balls).

- $K=K^{+} \cup_{S} K^{-}$, where $K^{ \pm} \subset B^{ \pm}$( $S$ cuts $K$ into over bridges and under bridges).

- $K^{ \pm}=K_{1}^{ \pm} \cup K_{2}^{ \pm} \cup \ldots K_{n}^{ \pm}$( $K$ is presented as $n$ over bridges and $n$ under bridges).

- $D^{ \pm}=D_{1}^{ \pm} \cup D_{2}^{ \pm} \cup \ldots D_{n}^{ \pm}\left(\right.$each $K_{i}^{ \pm} \cup p\left(K_{i}^{ \pm}\right)$bounds a disk $D_{i}^{ \pm}$such that $\left.p\left(D_{i}^{ \pm}\right)=p\left(K_{i}^{ \pm}\right)\right)$.

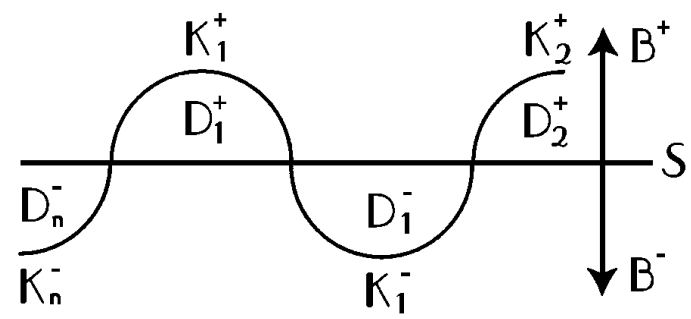

Figure 1. View from level surface

We take $n$ minimal over all bridge presentations of $p(K)$.

Lemma 2.2. We may assume that:

(a) $F \cap D^{-}=\emptyset$,

(b) $F \cap B^{-}$consists of disks,

(c) $F \cap D^{+}$consists of arcs, and

(d) any component of $F \cap B^{+}-D^{+}$is a disk.

Proof. (a): Simply push out $F$ near $D^{-}$into $B^{+}$.

(b): If there exists a component of $F \cap B^{-}$which is not a disk, then $F \cap B^{-}$ has a compressing disk $E$ in $B-N\left(D^{-}\right)$since $B-N\left(D^{-}\right)$is a 3-ball. By the incompressibility of $F$ in $S^{3}-K, \partial E$ bounds a disk in $F$. Then by cutting and pasting $F$ along $E$, we have a new incompressible surface $F^{\prime}$ and a sphere $F^{\prime \prime}$. Replace $F$ with $F^{\prime}$ and continue this operation.

(c): Suppose there exists a loop component of $F \cap D^{+}$and let $E$ be an innermost disk in $D^{+}$. Then the similar argument to (b) passes by using $E$. 
(d): If there exists a component of $F \cap B^{+}-D^{+}$which is not a disk, then $F \cap B^{+}-D^{+}$has a compressing disk $E$ in $B^{+}-D^{+}$. By using $E$, we can show (d) similarly.

We take a 2-tuple lexicographically ordered complexity measure $\left(\left|F \cap B^{-}\right|\right.$, $\left.\left|F \cap D^{+}\right|\right)$minimal. Note that the complexity measure is not $(0, *)$. For $(0, *)$, $F$ fails to be incompressible in $S^{3}-K$ since $\left(B^{+}, K^{+}\right)$is a trivial tangle. If the complexity measure is $(1,0)$, then we have the conclusion $(2)$.

Hereafter, we suppose that the complexity $\left(\left|F \cap B^{-}\right|,\left|F \cap D^{+}\right|\right) \geq(1,1)$.

Then we obtain a connected graph $G$ in $F$ by regarding $F \cap B^{-}$and $F \cap D^{+}$as vertices and edges respectively. Note that every vertex has a positive even valency by the construction.

An arc $\alpha_{j}$ of $F \cap D_{i}^{+}$divides $D_{i}^{+}$into two disks $\delta_{j}$ and $\delta_{j}^{\prime}$, where $\delta_{j}^{\prime}$ contains $K_{i}^{+}$. Put $\beta_{j}=\delta_{j} \cap S$. We may assume that $p\left(\alpha_{j}\right)=p\left(\delta_{j}\right)=\beta_{j}$ for all $\alpha_{j}$. We assign an orientation endowed from $K_{i}$ to $\alpha_{j}$ and $\beta_{j}$ naturally (see Figure 2).

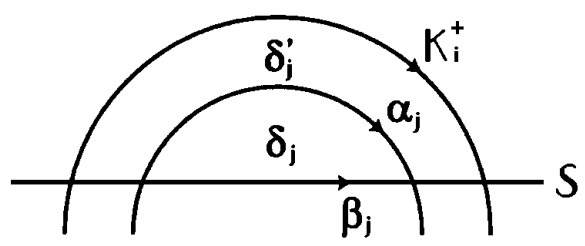

Figure 2. $\alpha_{j}$ and $\beta_{j}$ have the orientation

Lemma 2.3. For any arc $\alpha_{j}$ of $F \cap D_{i}^{+}, \beta_{j} \cap p\left(K^{-}\right) \neq \emptyset$.

Proof. Suppose that there exists an arc $\alpha_{j}$ of $F \cap D_{i}^{+}$such that $\beta_{j} \cap p\left(K^{-}\right)=\emptyset$. By exchanging $\alpha_{j}$ if necessary, we may assume that $\alpha_{j}$ is outermost in $D_{i}^{+}$, that is, int $\delta_{j} \cap F=\emptyset$. If $\alpha_{j}$ connects different vertices, then a $\partial$-compression of $F$ along $\delta_{j}$ reduces the complexity. Otherwise, $\alpha_{j}$ incidents a single vertex, say $D_{k}^{-}$. We perform a $\partial$-compression of $F$ along $\delta_{j}$, and obtain an annulus $A$ consisting of the disk $D_{k}^{-}$and the resultant band $b$. Since we chose an outermost arc $\alpha_{j}$ and $\beta_{j} \cap p\left(K^{-}\right)=\emptyset$, there exists a compressing disk for $A$ in $B^{-}-K^{-}$. By retaking $F$ along the compressing disk, we can reduce the complexity. In both cases, there is a contradiction in the assumption the complexity is minimal.

Now we pay attention to a face $f$ of $G$ in $F$. A corner is a subarc of $\partial(F \cap$ $\left.B^{-}\right)-\left(F \cap D^{+}\right)$. The cycle $\partial f$ for $f$ is a loop consisting of edges and corners such that it bounds $f$. The edges have orientations as previously mentioned.

Lemma 2.4 (The cycle lemma). For any face $f$, the cycle $\partial f$ can not be oriented. 
Proof. Suppose that there is a face $f$ such that $\partial f$ can be oriented. Then, since no corner of $\partial f$ intersects $p(K)$, and by Lemma 2.3, $p(\partial f)$ has non-zero intersection number with $p\left(K^{-}\right)$on $S$. Figure 3 illustrates the projection of $f$ and $K^{-}$on $S$. This is a contradiction.

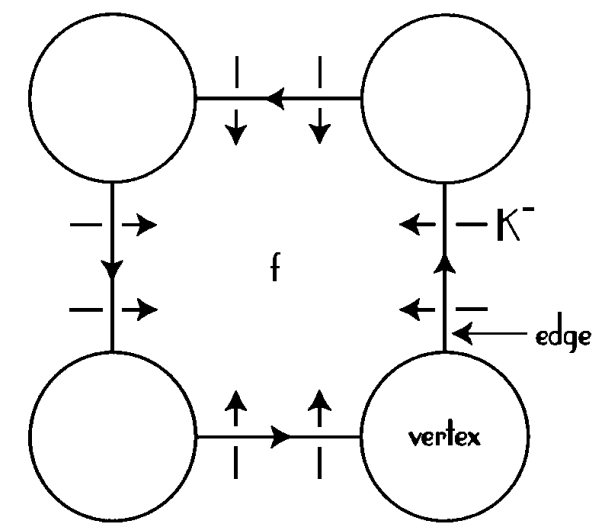

Figure 3. $p(\partial f)$ has non-zero intersection number

For each face $f$ of $G$ and any point in the interior of any edge of $\partial f$, we can find an arc $\gamma$ on $f$ satisfying the following property.

$\left.{ }^{*}\right) \gamma$ connects two edges of $\partial f$ whose orientations are different in $\partial f$.

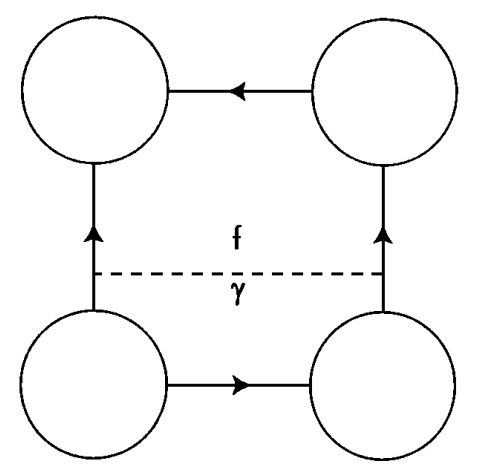

Figure 4. $\gamma$ with the property $(*)$

Lemma 2.4 assures the existence of such an $\operatorname{arc} \gamma$. 
To find a loop $l$ on $F$ with $l k(l, K) \neq 0$, we depart a point in the interior of any edge of $G$, trace arcs with the property $(*)$, and will arrive at the face on which we have walked. Connecting these arcs, we will obtain an oriented loop $l$ in $F \cap B^{+}$ with a suitable orientation such that $l$ has a positive intersection number with edges of $G$ on $F$. Thus we got an oriented loop $l$ in $F$ which has non-zero linking number with $K$. Since any loop in a splitting sphere is contractible in $S^{3}-K$, we have the conclusion (1).

This completes the proof of Theorem 2.1.

\section{Proof of Theorem 1.4}

Let $K$ be a positive knot or link in $S^{3}$ and $F$ be a decomposing sphere for $K$. We put $K$ and $F$ as the proof of Theorem 2.1 except that two points $p_{1}$ and $p_{2}$ of $F \cap K$ are in int $B^{+}$or int $B^{-}$. Note that $p_{1}$ and $p_{2}$ can not be the ends of a single arc of $F \cap D^{ \pm}$because the tangle $\left(B^{ \pm}, K^{ \pm}\right)$is trivial and $F$ is a decomposing sphere. Hence, there are two $\operatorname{arcs} e_{1}$ and $e_{2}$ of $F \cap D^{ \pm}$whose ends contain $p_{1}$ and $p_{2}$ respectively. We deform $F$ by an isotopy relative to $K$ so that $p\left(e_{i}\right)=p\left(p_{i}\right)$ $(i=1,2)$. We take the number of bridges $n$ minimal.

Thus we have the following data in addition to the data in the proof of Theorem 2.1 .

- $F \cap K=p_{1} \cup p_{2} \subset \operatorname{int} B^{ \pm}$

- $F \cap D^{ \pm} \supset e_{i} \supset p_{i}(i=1,2)$.

- $p\left(e_{i}\right)=p\left(p_{i}\right)(i=1,2)$.

Lemma 3.1. We may assume that:

(a) $F \cap D^{-} \subset e_{1} \cup e_{2}$,

(b) $F \cap B^{-}$consists of disks,

(c) $F \cap D^{+}$consists of arcs, and

(d) any component of $F \cap B^{+}-D^{+}$is a disk.

Proof. This can be done by an isotopy of $F$ since Theorem 1.3 assures us that $S^{3}-K$ is irreducible.

We take a 2-tuple lexicographically ordered complexity measure $\left(\left|F \cap B^{-}\right|, \mid(F \cap\right.$ $\left.\left.D^{+}\right)-\left(e_{1} \cup e_{2}\right) \mid\right)$ minimal. Then we obtain a connected graph $G$ in $F$ by regarding $F \cap B^{-}$and $\left(F \cap D^{+}\right)-\left(e_{1} \cup e_{2}\right)$ as vertices and edges respectively. Corners of each face of $G$ may contain two points $\partial e_{1}-p_{1}$ and $\partial e_{2}-p_{2}$. Note that the complexity measure is not $(0, *)$, otherwise $F$ is not a decomposing sphere since $\left(B^{ \pm}, K^{ \pm}\right)$is a trivial tangle. If the complexity measure is $(1,0)$, then $F \cap S$ gives a desired loop since $p\left(e_{i}\right)=p\left(p_{i}\right)(i=1,2)$.

Lemma 3.2. For any arc $\alpha_{j}$ of $\left(F \cap D^{+}\right)-\left(e_{1} \cup e_{2}\right), \beta_{j} \cap p\left(K^{-}\right) \neq \emptyset$. 
Proof. This can be done by the same argument to Lemma 2.3.

Hereafter, we assume that $\tilde{K}$ is prime.

Lemma 3.3. There is no vertex of $G$ with valency 1.

Proof. Suppose that there is a vertex $V$ with valency 1 . Then only one edge $\alpha$ incident to $V$, and hence exactly one of $e_{1}$ and $e_{2}$ is attached to $V$ or contained in $V$. Thus $\partial V$ intersects $\tilde{K}$ in two points. Since $\tilde{K}$ is prime, $\partial V$ bounds a disk $E$ in $S$ which intersects $p(K)$ in an unknotted arc. In the former case, $p(K) \cap E$ lies under a subarc of $K^{+}$by the minimality of the number of bridges $n$. Then by an isotopy of $F$ along the 3-ball which is bounded by $V \cup E$, we can reduce the complexity. See Figure 5. In the latter case, $E$ intersects $K$ in one point, and $V \cup E$ bounds a pair of a 3-ball and an unknotted subarc of $K^{-}$by the minimality of $n$. Then an isotopy of $F$ along the pair can reduce the complexity. See Figure 6 . In both cases, there is a contradiction in the assumption the complexity is minimal.

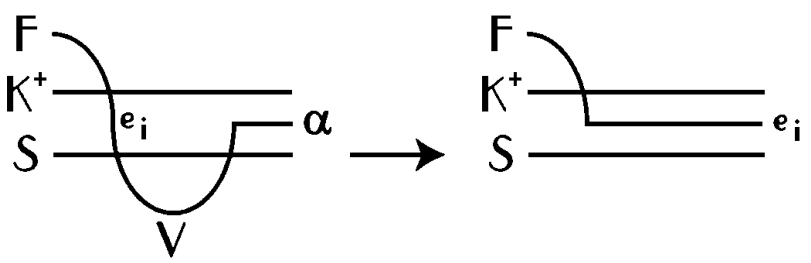

Figure 5. Isotopy of $F$ along the 3-ball

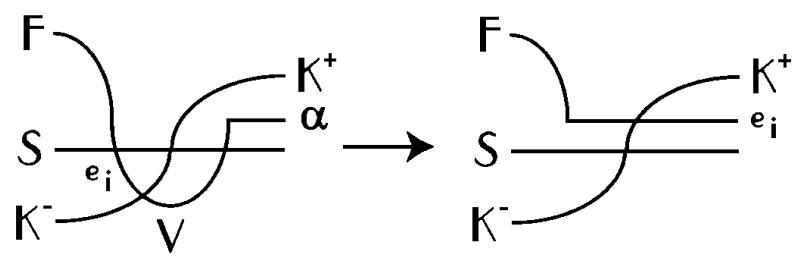

Figure 6. Isotopy of $F$ along the pair

Lemma 3.4. There is no face $f$ of $G$ in $F$ such that $\partial f$ is a loop of $G$. 
Proof. Suppose there exists a face $f$ as Lemma 3.4. Then $\partial f$ consists of an edge $\alpha$ of $G$ and a subarc $\gamma$ of the boundary of a vertex $V$ of $G$. By Lemma 3.2, $p(\alpha)$ intersects $p\left(K^{-}\right)$. Moreover, since the loop $\gamma \cup p(\alpha)$ bounds a disk $E$ in $S$, $\left|p(\alpha) \cap p\left(K^{-}\right)\right|=1$ and $\gamma$ meets exactly one of $e_{1}$ and $e_{2}$, say $e_{1}$. Thus a loop $l=\partial N(\partial E ; E)-\partial E$ intersects $\tilde{K}$ in two points. Since $\tilde{K}$ is prime, int $E$ intersects $p(K)$ in an embedded arc. Then, there are two possibilities for $e_{1}, e_{1} \subset f$ or $e_{1} \subset V$. In the former case, $f \cup E$ bounds a pair of a 3-ball and an unknotted arc, and an isotopy of $F$ along the pair eliminates $\alpha$. In the latter case, $f \cup E$ bounds a 3-ball, and an isotopy of $F$ along the 3-ball eliminates $\alpha$. These contradict the minimality of the complexity.

Hence we have a condition that:

- $G$ has at least two vertices,

- every vertex has valency at least two, and

- all faces of $G$ in $F$ are disks.

Next, we pay attention to a face of $G$ in $F$.

Lemma 3.5. For any face $f$, the cycle $\partial f$ can not be oriented.

Proof. If all corners of $f$ do not meet $e_{1} \cup e_{2}$, then this is same to Lemma 2.4.

If exactly one corner of $f$ meets $e_{1}$ or $e_{2}$ at one point, then $f$ and some $K_{i}^{+}$have the intersection number \pm 1 , or a vertex which meets $f$ along the corner intersects some $K_{k}^{-}$in one point. Since $p(\partial f)$ and $p\left(K^{-}\right) \cap p\left(K_{i}^{+}\right)$must have the intersection number zero, $\partial f$ is bounded by a loop of $G$ consisting of a vertex and an edge $\alpha$, and $p(\alpha)$ intersects $p\left(K^{-}\right)$in one point. Then Lemma 3.4 gives the conclusion.

If some corners of $f$ meet both $e_{1}$ and $e_{2}$, then the corners of $f$ have the intersection number zero with $p(K)$ because $F$ and $K$ have the intersection number zero. In such a situation, we have a contradiction same as the proof of Lemma 2.4 .

By Lemma 3.5, starting a face $f$ of $G$ in $F$ whose closure is a disk, we can get a loop $l$ in $F-K$ with $|l k(l, K)| \geq 2$. But this is impossible because any loop in $F-K$ is null-homotopic in $S^{3}-K$ or has linking number \pm 1 with $K$. This finishes the proof of Theorem 1.4.

Acknowledgments. The author would like to thank Hiroshi Matsuda, Koya Shimokawa and the referee for heartful comments.

\section{References}

[1] R. J. Aumann, Asphericity of alternating knots, Ann. of Math. 64 (1956), 374-392.

[2] P. R. Cromwell, Homogeneous links, J. London Math. Soc. (2) 39 (1989), 535-552. 
[3] P. R. Cromwell, Positive braids are visually prime, Proc. London Math. Soc. (3) 67 (1993), 384-424.

[4] W. Menasco, Closed incompressible surfaces in alternating knot and link complements, Topology 23 (1984), 37-44.

[5] M. Ozawa, Synchronism of an incompressible non-free Seifert surface for a knot and an algebraically split closed surface in the knot complement, Proc. Amer. Math. Soc. 128 (2000), 919-922.

[6] T. Tsukamoto, A criterion for almost alternating links to be non-splittable, preprint.

Makoto Ozawa

Waseda University

School of Education

Department of Mathematics

Nishiwaseda 1-6-1

Shinjuku-ku

Tokyo 169-8050

Japan

e-mail: ozawa@musubime.com

(Received: June 28, 2000)
Current address:

Komazawa University

Faculty of Letters

Natural Science Faculty

1-23-1 Komazawa, Setagaya-ku

Tokyo, 154-8525

Japan

(2) To access this journal online:

(20) http://www.birkhauser.ch 